\title{
Estilo de vida en pacientes adultos con Diabetes mellitus tipo $\mathbf{2}^{\mathbf{1}}$
}

\author{
Pedro César Cantú Martínez²
}

Institución: Universidad Autónoma de Nuevo León

\section{COMO CITAR}

Cantú, P.C.(2014). Estilo de vida en pacientes adultos con Diabetes mellitus tipo 2. Rev. Enfermería Actual en Costa Rica, 27, 1-14. DOI: http://dx.doi.org/10.15517/revenf.v0i27.15996

\section{RESUMEN}

Introducción. Se examina y describe el estilo de vida de pacientes con Diabetes mellitus tipo 2, los cuales asistieron al control de diabetes en diferentes Centros de Salud Urbanos del Área Metropolitana de Monterrey, N.L., México, para estudiar los determinantes individuales y establecer la relación entre el estilo de vida evaluado y el percibido.

Método. La muestra fue no aleatoria, seleccionada por conveniencia, en el orden de asistencia a consulta. La población fue de 65 pacientes, que contaron con características muy similares, de acuerdo con el diagnóstico de diabetes mellitus tipo 2 y que accedieron voluntariamente a participar, en el periodo julio-septiembre del año 2012. Se utilizó el instrumento denominado IMEVID, para explorar el estilo de vida y se relacionó con la percepción de los pacientes y sus dimensiones.

Resultado. Se colige que un 29,23\% consideraba tener un "buen estilo de vida", porcentaje que correspondió a una clasificación "saludable", mientras que la mayor concentración se tiene un inadecuado estilo de vida (70,77\%), con dos clasificaciones de los participantes; luego, un 56,92\% se caracteriza por una conducta "moderadamente saludable" y solamente el 13,85\% de estos se catalogó como "poco saludable". Se detectó una correlación significativa entre el estilo de vida evaluado y las dimensiones consideradas, excepto con el estado emocional. Asimismo, se estableció una relación entre el estilo de vida evaluado y el percibido por los participantes.

Conclusión. El paulatino esclarecimiento del concepto denominado "estilo de vida" ha permitido redimensionar la noción del estado de "salud-enfermedad". Los resultados concuerdan con otras investigaciones desarrolladas en México.

Palabras clave: adultos; Diabetes-mellitus; estilo-de-vida; evaluación.

\footnotetext{
${ }^{1}$ Fecha de recepción: 12 de marzo del 2014

Fecha de aceptación: 14 de julio del 2014

${ }^{2}$ Universidad Autónoma de Nuevo León, Facultad de Salud Pública y Nutrición, México. Correo electrónico: pedro.cantum@uanl.mx
} 


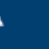

,

$=$

\section{Revista Electrónica Entermeria actual en costa Rica}

www.revenf.ucr.ac.cr

\section{Lifestyle in patients with type 2 Diabetes mellitus ${ }^{1}$}

Pedro César Cantú Martínez ${ }^{2}$

Institution: Autónoma de Nuevo León University

\section{CITED:}

\begin{abstract}
Background: it examines and describes the style of life in patients with type 2 diabetes mellitus, who attended the diabetes control in different Health Centers of Monterrey Metropolitan Area, N.L., Mexico, to study individual determinants and establish the relationship between style rated life and perceived. The sample was not randomly selected by convenience, in order to assist the inquiry.

Method: the population was 65 patients that counted with very similar characteristics, according to the diagnosis of type 2 diabetes mellitus and voluntarily agreed to participate, from July to September 2012. Instrument known as IMEVID was used to explore the style life and was related to the perception of patients and their dimensions. Result: the 29,23\% said after the instrument has been applied to sustain a "good lifestyle", which corresponded to a "healthy" rating, while the highest concentration was found in an inappropriate lifestyle (70,77\%), with two classifications of participants, 56,92\% was found with a "moderately healthy" and only 13,85\% of these are catalog as "unhealthy" behavior. Significant correlation between the styles of life evaluated and considered dimensions, except with the emotional state was found. Relationship existed between the style of life evaluated and perceived by patients.

Conclusion: the gradual clarification of the concept known as "lifestyle" has enabled the health sciences resize the notion of the state of "health and disease"; results are consistent with other studies conducted in Mexico.
\end{abstract}

Key words: adult; Diabetes-mellitus; evaluation; life-style.

\footnotetext{
${ }^{1}$ Date of receipt: March 12, 2014

Date of acceptance: July 14, 2014

2 Autónoma de Nuevo León University, Faculty of Public Health and Nutrition, México. E-mail: pedro.cantum@uanl.mx
} 


\section{INTRODUCCIÓN}

La Diabetes mellitus crece con tal rapidez que se le considera un problema que aqueja a la población con mayúsculas cargas de carácter médico, social y económico en el mundo, las cuales socavan los beneficios del desarrollo alcanzados hasta el momento por numerosos naciones de ingresos bajos y medios, por lo tanto, atenta contra la sostenibilidad socioeconómica y el propio desarrollo humano de las sociedades (Federación Internacional de Diabetes, 2011). El padecimiento de Diabetes mellitus es advertido por la Organización Mundial de la Salud (2012) como un reto de carácter internacional, puesto que se deduce que en el orbe coexisten más de 347 millones de personas con dicha enfermedad y es factible que este monto se duplique para el año 2030. Este vertiginoso aumento en la prevalencia de tal padecimiento en el contexto internacional ha confirmado la relación entre el estilo de vida y el desarrollo de este padecimiento.

Particularmente, en México la Diabetes mellitus es una de las principales causas de morbimortalidad, cuyo incremento ha producido aproximadamente 60 mil muertes y 400000 casos nuevos al año (Lerman, 2007; Secretaría de Salud, 2008), al punto de que se considera que el 90\% de los casos de Diabetes mellitus tipo 2 se deben al estado de malnutrición en el que se incluye el sobrepeso y la obesidad (Secretaría de Salud, 2010). De acuerdo con la Encuesta Nacional de Salud y Nutrición en México, la cifra de casos con diabetes mellitus o en riesgo de poseerla ha crecido entre los años 2002 y 2012, periodo donde se observaba una prevalencia de Diabetes mellitus en adultos del 4,6\% que alcanzó al 9,2\% de la población mexicana (Gutiérrez et al, 2012).

Dado lo anterior, en México la Diabetes mellitus se constituye en un desafío para las instituciones del sector salud del país, debido a que este padecimiento, como otras afecciones crónicas, son consecuencia de estilos de vida poco saludables, entre cuyos determinantes sociales, además de la predisposición genética, se encuentran las costumbres de alimentación, sedentarismo, el consumo de alcohol y tabaco, como promotoras de su desarrollo (Unwin, Roglic y Whiting, 2010). En cuanto al estilo de vida, Wilson y Ciliska (1984) lo definen como la confluencia de un grupo de determinaciones individuales que llegan a aquejar la salud y sobre las cuales se ejerce cierta condición de dominio voluntario y consciencia por parte de las personas. En el presente estudio se explora descriptivamente el estilo de vida de pacientes con Diabetes mellitus tipo 2, así como las particularidades que revelan y la relación con el estilo de vida evaluado respecto del percibido.

\section{MATERIALES Y MÉTODO}

La población de estudio estuvo integrada por 65 participantes, los cuales contaron con características muy similares en relación con el diagnóstico de Diabetes mellitus tipo 2.

En lo referente a la investigación, es de tipo descriptivo y transversal, cuyo propósito fue reconocer el estilo de vida de los pacientes con Diabetes mellitus tipo 2 que asistieron al control de dicha enfermedad en diferentes Centros de Salud Urbanos del Área Metropolitana de Monterrey, N.L., México, durante el periodo de julio a septiembre del 2012.

La evaluación se realizó con el instrumento IMEVID, con el que se exploró el estilo de vida de los pacientes con Diabetes mellitus tipo 2, que cuenta con un alfa de Cronbach de 0,81 (López, Ariza, Rodríguez y Munguía, 2003). Este instrumento está constituido por 25 preguntas, que abarcan distintas dimensiones con tres 
opciones para contestar, con estimaciones puntuales cuyos valores son 4,2 y 0 puntos: el valor más alto corresponde a la mejor condición de evaluación y la menor, al peor estado de valoración. Las dimensiones que comprende este instrumento son prácticas alimentarias, actividad física, conocimiento sobre el padecimiento, consumo de tabaco y alcohol, estado emocional y la adherencia al tratamiento. La catalogación de los pacientes, respecto de su estilo de vida, se realizó conforme a la siguiente progresión numérica: 100-75, 74-50, 49-25 y 24-0 (Ramírez, et al, 2011), las cuales fueron designadas en dos categorías con cuatro clasificaciones: un buen estilo de vida, que representa una conducta saludable; inadecuado estilo de vida, que involucra conductas moderadamente saludable, poco saludable y no saludable, respectivamente. Luego de la evaluación, se relacionó con la percepción del estilo de vida aportado por los pacientes, al cuestionarles cómo percibían su estilo de vida, ya fuera muy saludable, saludable y no saludable. Para el procesamiento y análisis de la información recabada se utilizó el programa SPSS v.21.

\section{Consideraciones éticas}

Los participantes accedieron espontáneamente a participar en el estudio de acuerdo con el consentimiento informado y el marco legal vigente en México en materia de investigación en salud que la cataloga como "sin riesgo" (EUM, 1986).

\section{RESULTADOS}

\section{a. Características sociodemográficas y condiciones de salud de la población de estudio}

En el presente estudio participaron un total de 65 pacientes: el mayor porcentaje de ellos son del género femenino $(f=47,72,3 \%)$, la edad de mayor asiduidad encontrada en el grupo de estudio correspondió al intervalo de 40 a 59 años $(f=37,56,9 \%)$; según el estado civil revelado por los participantes, la mayor reiteración correspondió a casado $(f=45,69,2 \%)$. Por otra parte, se colige que la ocupación mayormente realizada por parte de pacientes es la realización de tareas en el hogar $(f=35,53,8 \%)$, en lo alusivo a la escolaridad de la población de estudio, el mayor porcentaje incumbió primaria incompleta $(f=32,49,2 \%)$ (véase tabla 1$)$. 
Tabla 1

Monterrey, Nuevo León: Distribución de las características generales de la población seleccionada con Diabetes mellitus tipo 2

\begin{tabular}{lcc}
\hline Variables & Frecuencia & Porcentaje \\
\hline Sexo & 47 & 72,3 \\
Mujeres & 18 & 27,6 \\
Hombres & & \\
& & \\
Edad (años) & 12 & 18,4 \\
Menores de 40 & 37 & 56,9 \\
40 a 59 & 16 & 24,6 \\
Mayores de 60 & & \\
& & \\
Estado civil & 4 & 6,1 \\
Soltero & 45 & 69,2 \\
Casado & 8 & 12,3 \\
Divorciado & 8 & 12,3 \\
Unión Libre & & \\
& & \\
Ocupación & 35 & 53,8 \\
Labores en el hogar & 28 & 43,0 \\
Trabajador activo & 2 & 3,0 \\
Pensionado & & \\
& & \\
Escolaridad & & \\
Primaria incompleta & 32 & 49,2 \\
Primaria completa & 69,2 \\
Secundaria completa & 6,2 \\
Bachillerato completo & Profesional & 6,1 \\
n= 65 & 4 & 6,1 \\
& & \\
\hline & & \\
\hline
\end{tabular}

Fuente: elaboración propia

En cuanto a las particularidades de salud de los participantes, al mayor porcentaje se les diagnosticó sobrepeso $(f=36,55,3 \%)$; los participantes declararon mayormente que el tiempo de evolución del padecimiento fue menor a 5 años $(f=40,61,5 \%)$ y el tipo de tratamiento que más se reiteró para su control fue el uso de antidiabéticos orales $(f=52,80 \%)$, como la combinación de metformina y glibenclamida. Respecto de la enfermedad concomitante, el 70,7\% ( $f=46)$ padece hipertensión arterial y cuando se les cuestionó acerca de su percepción sobre el estilo de vida que llevan posterior al diagnóstico de Diabetes mellitus tipo 2, el 75,3\% ( $f=49$ ) lo consideró como muy saludable (véase tabla 2). 
Tabla 2

Monterrey, Nueva León: Condiciones de salud de la población de estudio con Diabetes mellitus tipo 2

\begin{tabular}{lcc}
\hline Variables & Frecuencia & Porcentaje \\
\hline Evaluación nutricional & 25 & \\
Obesidad & 36 & 38,4 \\
Sobrepeso & 4 & 55,3 \\
Normal & & 6,1 \\
Tiempo de evolución de la Diabetes mellitus tipo 2 & 40 & 61,5 \\
Menor o igual a 5 años & 9 & 13,8 \\
6 a 10 años & 16 & 24,6 \\
Mayor de 10 años & & \\
Tratamiento de la Diabetes mellitus tipo 2 & 52 & 80,0 \\
Antidiabéticos orales & 4 & 6,1 \\
Insulina & 4 & 6,1 \\
Antidiabéticos orales más insulina & 4 & 6,1 \\
Dieta & & \\
Enfermedades concomitantes & 46 & 70,7 \\
Hipertensión arterial & 19 & 29,2 \\
No reportó & & \\
Estilo de vida percibido por los participantes & 7 & 10,7 \\
No saludable & 9 & 13,8 \\
Moderadamente saludable & 49 & 75,3 \\
Saludable & & \\
Estilo de vida evaluado en los participantes & 9 & 13,8 \\
Poco saludable & 37 & 56,9 \\
Moderadamente saludable & 19 & 29,2 \\
Saludable \\
n= 65
\end{tabular}

Fuente: elaboración propia

\section{b. Evaluación del estilo de vida}

De la población estudiada, 65 participantes, el 29,2\% $(f=19)$ manifestó que tras haber aplicado el instrumento, afirmó experimentar un "buen estilo de vida" que corresponde a una clasificación "saludable", mientras que la mayor concentración se ubica en un inadecuado estilo de vida $(f=46,70,7 \%)$, con dos clasificaciones de los participantes; el 56,9\% ( $f=37)$ se presentó una conducta "moderadamente saludable" y solamente el $13.8 \%(f=9)$ de estos se catalogó como "poco saludable" (véase tabla 2). Se obtuvo calificaciones de IMEVID que fluctuaron entre 34 y 82, con un promedio de 64 y una variación de los datos respecto del valor promedio reportado de 12,3, cantidad que indica que la población estudiada cuenta con un estilo de vida promedio clasificada como "moderadamente saludable". 


\section{c. Dimensiones}

\section{c.1 Prácticas alimentarias}

En lo referente a las prácticas alimentarias llevadas a cabo por los participantes en la investigación, se reveló que el 36,92\% $(f=24)$ cuenta con un "buen estilo de vida" y un patrón de conducta "saludable", mientras que el $63 \%$ de los participantes se clasificó dentro de un "inadecuado estilo de vida" . Al respecto, se observó que el 41,5\% $(f=27)$ de los participantes practica hábitos "moderadamente saludables", mientras que el 21,5\%, $(f=14)$ corresponde a un modo "poco saludable". Se observaron calificaciones de IMEVID que fluctuaron entre 33 y 94 , con un promedio de 67 y una variación de los datos de 17,4, cifra de la que es correcto afirmar que la población investigada cuenta con un estilo de vida promedio "moderadamente saludable" en lo que respecta a sus prácticas alimentarias.

\section{c.2 Actividad física}

En cuanto a la actividad física realizada por los pacientes en el estudio, las indagaciones mostraron que el $20 \%(f=13)$ cuenta con un "buen estilo de vida" y una clasificación "saludable", sin embargo, el $80 \%(f=52)$ muestra un "inadecuado estilo de vida", lo cual se refleja en un 47,6\% $(f=31)$,"moderadamente saludable"; un 20\% ( $\mathrm{f}=13)$, es "poco saludable" y 12,3\% $(f=8)$, "no saludable". Se averiguó que las evaluaciones de IMEVID oscilaron entre 0 y 100 , con un promedio de 53 y una variación de 25 , resultado que evidencia un estilo de vida promedio considerado como "moderadamente saludable" para dicha población.

\section{c.3 Consumo de tabaco y alcohol}

En lo que respecta al consumo de tabaco y alcohol, se evidencia que, en relación con el consumo de tabaco, el 92,3\% $(f=60)$ de las personas estudiadas no consumen tabaco, por lo cual cuentan con una clasificación de "saludable" en el marco de un "buen estilo de vida", mientras que tan solo el 7,6\% ( $f=5)$ de dicho grupo se clasifica como "moderadamente saludable", que corresponde a un "inadecuado estilo de vida". Se percibió que las estimaciones de IMEVID oscilaron entre 50 y 100, con un promedio de 97 y una variación de 12,3, cifra que respecto del consumo de tabaco, manifiesta que la población cuenta con estilo de vida promedio, considerada como "saludable", entendido como "no fuma mayormente". En cuanto al consumo de alcohol, el 80\% $(f=52)$ no lo consumen, de modo que pertenecen a la clasificación de "saludable", es decir, un "buen estilo de vida"; luego, se identificó que el 20\% ( $f=13)$, practica un "inadecuado estilo de vida" en el que se clasifican un $4,6 \%(f=3)$ de los participantes en un estado "moderadamente saludable", sin embargo, en la categoría de "poco saludable" y "no saludable" se detectó un $12,3 \%(f=8)$ y un $3 \%(f=2)$ de la población estudiada respectivamente. Se apreció que los valores de IMEVID variaron entre 0 y 100, con un promedio de 85 y una variación de 20, lo cual, en lo que se refiere al consumo de alcohol, evidencia que la población cuenta con estilo de vida promedio, estimada como "saludable".

\section{c.4 Conocimiento sobre el padecimiento}

En lo relativo a acudir a grupos o profesionales para obtener un mayor conocimiento sobre su enfermedad, se averiguó que el 30,7\% $(f=20)$ de los participantes en la investigación, cuenta con una clasificación de 
"saludable" en el contexto de un "buen estilo de vida", mientras que el 26,1\% (f=17) se es "moderadamente saludable"; mientras que en la condición de "poco saludable" y "no saludable" están el 23\% $(f=15)$ y 20\% $(f=13)$ de los participantes respectivamente que, en conjunto, suman un 69,2\% $(f=45)$ dentro de un "inadecuado estilo de vida". Se distinguieron calificaciones de IMEVID que fluctuaron entre 0 y 100, con un promedio de 47 y una variación de los datos de 34,7, cifra de la que se colige que la población indagada cuenta con estilo de vida promedio "poco saludable", en otras palabras, carece de interés por informarse acerca de su padecimiento.

\section{c.5 Estado emocional}

En lo referente al estado emocional en el que se encontraban los participantes en el estudio, un 16,9\% $(f=12)$ se incluyó dentro de la clasificación de "saludable", es decir, cuenta con un "buen estilo de vida", sin embargo, el $83 \%(f=54)$ se enmarca en un "inadecuado estilo de vida", del que un 58,4\% ( $f=38)$ presentaba un estado "moderadamente saludable", $10,7 \%(f=7)$ y un $13,8 \%(f=9)$ manifestó un estado "poco saludable" y "no saludable" comparativamente. Se examinó que las evaluaciones de IMEVID oscilaron entre 0 y 100 , con un promedio de 53 y una variación de 23,2, lo cual, respecto de esta dimensión, evidencia que la población cuenta con un estilo de vida promedio, considerada como "moderadamente saludable"; por tanto, no cuenta con una razonable estabilidad emocional.

\section{c.5 Adherencia al tratamiento}

En relación con el apego al tratamiento, el estudio expresó los participantes se encontraban en una clasificación de "saludable": el 61,5\% $(f=40)$, un "buen estilo de vida", mientras que un 29,2\% $(f=19)$ fue clasificado como "moderadamente saludable". Por otra parte, en la clasificación de "poco saludable" y "no saludable" se detectó un 4,62\% $(f=3)$ para cada una de estas codificaciones, que corresponden a un $38,4 \%(f=25)$ con un "inadecuado estilo de vida". Se observó que los valores de IMEVID oscilaron entre 0 y 100 , con un promedio de 76 y una variación de 25,5 , cifra de la que se concluye que la población cuenta con estilo de vida promedio, considerada como "saludable", en otras palabras, se refiere a si está adherida al tratamiento que se le asigna médicamente, para el control de su enfermedad.

\section{d. Correlación entre el IMEVID y las dimensiones evaluadas}

En lo que respecta al estilo de vida y su asociación con las dimensiones evaluadas, se obtuvo correspondencias estadísticamente significativas prácticamente con todas las dimensiones, excepto con el estado emocional del paciente (véase tabla 3). 
Tabla 3

Monterrey, Nuevo León: Correlación del IMEVID con las dimensiones que lo integran

IMEVID

Hábitos alimentarios

Actividad física

No consumo de tabaco

No consumo de alcohol

Conocimiento sobre la enfermedad

Estado emocional

Adherencia al tratamiento

$\mathrm{n}=65$

\section{Correlación Ro Sperman Significancia}

0.78

$<0.01$

0.352

$<0.01$

0.289

$<0.05$

0.494

$<0.01$

0.642

$<0.01$

0.229

0.066

0.755

Fuente: elaboración propia

e. Relación entre el estilo de vida evaluado y la percepción del estilo de vida de los pacientes

Al examinar la asociación entre el estilo de vida evaluado y la apreciación con que contaban los participantes acerca de sí en relación con la enfermedad, se identificó una relación significativa, de modo que se infiere que los participantes en la investigación manejan una adecuada percepción de su estado de salud en cuanto a la evolución de la enfermedad y los cambios en sus conductas (véase tabla 4).

\section{Tabla 4.}

Monterrey, Nuevo León: Distribución del estilo de vida evaluado versus estilo de vida percibido

\section{Población diabética estudiada}

Poco saludable

Estilo de vida evaluado
Estilo de vida percibido

No saludable Moderadamente saludable Saludable
Total
$4(6,1 \%)$
$1(1,5 \%)$
$4(6,1 \%)$
$9(13,8 \%)$

Moderadamente saludable

$2(3,0 \%)$

$8(12,3 \%)$

$27(41,5 \%) \quad 37(56,9 \%)$

Saludable

$1(1,5 \%)$

$0(0,0 \%)$

$18(27,6 \%) \quad 19(29,2 \%)$

Total

$7(10,7 \%)$

$9(13,8 \%)$

$49(75,3 \%) \quad 65(100 \%)$

Valor de $p: 0.002$

Fuente: elaboración propia

\section{DISCUSIÓN}

Con el paulatino esclarecimiento del concepto "estilo de vida", se ha redimensionó la noción del estado de "salud-enfermedad" que manifiesta un ser humano desde las ciencias de la salud, aparte de que permite reconocer que este binomio se encuentra circundado por determinantes que establecen dicha condición de manera particular. 


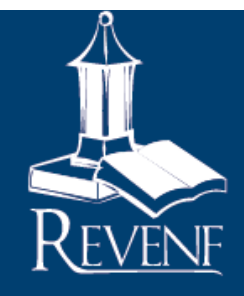

\section{Revista Electrónica Entermeria actual en costa Rica}

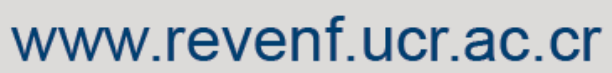

Sin duda, lo anterior propicia un mejor conocimiento de estos factores, de orden no biológico, que median en esa relación y que permiten esclarecer e interpretar la variación estructural vinculada con el proceso de búsqueda de la salud por cada persona (Coreil, Levin y Jacó, 1985; Espinosa, 2004); por consiguiente, el estilo de vida resulta de un entramado de decisiones de orden personal llevadas a cabo de forma consciente, las cuales tienen injerencia de forma franca en la salud de la persona (Wilson y Ciliska, 1984).

Particularmente, en las personas con diagnóstico Diabetes mellitus tipo 2, el estilo de vida se constituye en una variable reveladora del apego exitoso al tratamiento, que incluye una serie de medidas de carácter terapéutico y otras más relacionadas con patrones conductuales, así el recrudecimiento de los síntomas y estragos en el paciente son el efecto de una desatención a dichas pautas. En este orden de ideas, la población motivo de estudio manifestó encontrarse mayormente en un inadecuado estilo de vida $(70,7 \%)$, con una concentración principalmente en la clasificación de "moderadamente saludable" (56,9\%), lo cual se refiere a las dificultades por parte de los participantes para mantener un buen estilo de vida, que propicie una adherencia al tratamiento, con actitudes que les permitan superar los factores de orden personal que interfieren, para alcanzar exitosamente una conducta deseada y, en este caso, un estilo de vida adecuado y saludable (Ortíz y Ortíz, 2007). Los resultados de la presente investigación se comparan con otros estudios realizados, de lo que se observa que estadísticamente concuerdan en su comportamiento con Balcázar, Gurrola, Bonilla, Colín y Esquivel, (2008), Montejo (2009), Gómez, Avila y Candila (2012) y Tolosa, Candioti y D’Alessandro (2012) (véase anexo 1).

Por otra parte, Meneses, Ignacio, Mendoza, Moctezuma y Reyes de Jesús (2012), al referirse a dicho padecimiento, mencionan que en las naciones desarrolladas esta adherencia al tratamiento alcanza valores en el $50 \%$ de los casos, mientras que en México, estudios realizados muestran un apego del 54,2\% y 65\% (Durán, Rivera y Franco 2001; Meneses et al., 2012), muy similares al presente estudio en cuanto al apego al tratamiento en el $61,5 \%$ de los participantes.

Luego, en lo que respecta al comportamiento conductual de los pacientes diabéticos estudiados, de los valores de correlación significativos entre el estilo de vida evaluado con la gran mayoría de las dimensiones se ha observado que los participantes implementan cambios positivos en su estilo de vida con el objeto de poseer y conservar un buen estado de salud y evitar las complicaciones crónicas que se derivan y que median en esta patología, las cuales afectan de manera general la calidad de vida de quién la posee y acortan la expectativa de vida al empeorar el pronóstico funcional del paciente y sobrellevar consecuencias que pueden resultar en consecuencias letales.

No obstante, lo anterior no sucede con la dimensión emocional, particularidad que, según los autores, se debe a un estrés emocional notable al que se ven expuestos los pacientes diabéticos; esencialmente porque es un padecimiento que puede abreviar su periodo de vida y producirles inconvenientes graves, con cuadros de negación, incomodidad, ira o depresión (Garay, 2005). Lo anterior, aunado a las situaciones cotidianas, somete e impone una carga adicional al paciente que, de acuerdo con Bronfenbrenner (1979), se denomina mesosistema, el cual considera las relaciones de otros ambientes en los que el paciente diabético participa activamente, tales como la familia, el campo laboral y lo relativo al entorno social; tales entornos influyen y exacerban la condición del padecimiento en cuanto a no contar con una estabilidad emocional; por ende, no se relaciona con el estilo de vida evaluado en esta población. 
En cuanto a las dimensiones sobre el estilo de vida evaluado, cabe mencionar que los principales factores por considerar, de acuerdo con los resultados que manifiestan los participantes en el presente estudio, están relacionados con las prácticas alimentarias, la falta de actividad física y el estado emocional, de modo que conducen al paciente a vivir prácticas "moderadamente saludables". No obstante, en lo que se refiere a la relación con el conocimiento de su enfermedad, es notoria la falta o búsqueda de este conocimiento sobre la enfermedad, al punto de que los sitúa en un estado "poco saludable". Lo anterior se vincula con el grado de escolaridad que, según Bustos, Bustos, Bustos, Cabrera y Flores (2011), en nuestra población estudiada prevalece el de primaria incompleta, lo cual ubica a los participantes en un riesgo que puede agravar su padecimiento, dado que carecen de las capacidades para recibir la información y, por lo tanto, aprender lo suficiente sobre la enfermedad para crear un interés que, paulatinamente, los lleve a un cambio de conducta y los conduzca a mejorar su estilo de vida.

\section{CONCLUSIÓN}

El presente estudio permite abordar la enfermedad de la Diabetes mellitus tipo 2 desde varias aristas que constituyen las dimensiones de un estilo de vida de acuerdo con el IMEVID, de modo que posibilita conocer más acerca de este padecimiento a partir de los propios actores para conocer mejor sus posturas, así como los cambios actitudinales que implicarían una mejor restitución de la salud e integración social de quienes la padecen. Por último, se debe reconocer que clínica y socialmente, se originan factores predisponentes o precipitantes para la adopción de nuevas conductas positivas o la acentuación negativa de otras que ya existían visiblemente establecidas en los pacientes con Diabetes mellitus tipo 2.

\section{REFERENCIAS BIBLIOGRÁFICAS}

Balcázar, P., Gurrola, G., Bonilla, M., Colín, H.. y Esquivel , E. (2008). Estilo de vida en personas adultas con diabetes. Revista Científica Electrónica de Psicología, 6,147-158.

Bronfenbrenner, U. (1979). The ecology of Human Development. Cambridge: Harvard University Press.

Bustos, R., Bustos, A., Bustos, R., Cabrera, I. y Flores, J. (2011). Falta de conocimientos como factor de riesgo para ser hospitalizado en pacientes diabéticos tipo2. Archivos de Medicina Familiar, 13(1), 62-73.

Coreil, J., Levin, J. y Jaco, E. (1985). Life style an emergent concept in the sociomedical sciences. Cult Med Psychiatry, 9(4), 423-437.

Corona, J., Bryan, M. y Gómez, Y. (2008). Relación entre estilo de vida y control glicémico en pacientes con DM2. Revista Electrónica Ministerio de Salud, Marzo, 1-7.

Durán, B.R., Rivera, B. y Franco, E. (2001). Apego al tratamiento farmacológico en pacientes con diagnóstico de Diabetes mellitus tipo 2. Salud Publica Mex, 43, 233-236.

Espinosa, L. (2004). Cambios del modo y estilo de vida; su influencia en proceso de salud-enfermedad. Rev Cubana Estomtol, 41(3),1-6. 
Estados Unidos Mexicanos (EUM) (1986). Reglamento de la Ley General de Salud en Materia de Investigación en Salud. México. Diario Oficial de la Federación.

Federación Internacional de Diabetes (2011). Plan mundial contra la diabetes 2011-2021. Bruselas. FID. Recuperado de: http://www.idf.org/sites/default/files/attachments/GDP-Spanish.pdf

Garay, M. (2005). El paciente diabético y sus emociones. Memorias del II Encuentro de participación de la mujer en la ciencia. 19 al 20 de Mayo del 2005. León, Gto., México.

Gómez, P., Ávila, G. y Candila, J. (2012). Estilo de vida y control metabólico en personas con diabetes tipo 2 , Yucatán, México. Rev Enferm Inst Mex Seguro Soc, 20 (3), 123-129.

Gómez-Aguilar, P.I.S., Avila-Sansores, G.M. y Candila-Celis, J.A. (2012). Estilo de vida y control metabólico en personas con diabetes tipo 2, Yucatán, México. Rev Enferm Inst Mex Seguro Soc., 20 (3), 123-129.

Gutiérrez, J., Rivera, J., Shamah, T., Villalpando, S., Franco, A., Cuevas, L., Romero, M. y Hernández, M. (2012). Encuesta Nacional de Salud y Nutrición 2012. Resultados Nacionales. Cuernavaca, México D. F.: Instituto Nacional de Salud Pública (MX).

Lerman, I. (2007). La atención del paciente más allá del primer nivel de atención. Mesa Redonda XI: Hacia un modelo eficiente de atención al paciente diabético en México. XII Congreso de Investigación en Salud Pública. Edición Especial, Salud Pública Méx, 49, 99-103.

López, J., Ariza, C., Rodríguez, J., Munguía, C. (2003). Construcción y validación inicial de un instrumento para medir el estilo de vida en pacientes con Diabetes mellitus tipo 2. Salud Pública Méx, 45, 259-268.

Meneses, A., Ignacio, D., Mendoza, F., Moctezuma, M. y Reyes de Jesús, L. (2012). Factores que influyen en el abandono del tratamiento de Diabetes Mellitus tipo II en los usuarios del centro de salud de Tlacoachistlahuaca, Gro. Memorias del 2do Congreso Internacional de Pre-ALASRU 2012 "Diversidad y Contrastes en los Procesos Rurales en el Centro de México. Cuernavaca, Mor., México; 5 al 7 Septiembre del 2012, pp 1-10.

Montejo, M. (2009). Estilos de vida en diabéticos del Instituto Mexicano del Seguro Social: correlación con el modelo de Wallston. (Tesis de Maestría en Salud Pública). Universidad Veracruzana / Instituto de Salud Pública. Xalapa de Enríquez, Ver., México.

Organización Mundial de la Salud (2012). Diabetes. Nota descriptiva N ${ }^{\circ}$. 312. OMS. Recuperado de: http://www.who.int/mediacentre/factsheets/fs312/es/index.html.

Ortíz, M. y Ortíz, E. (2007). Psicología de la salud: Una clave para comprender el fenómeno de la adherencia terapéutica. Rev Méd Chile, 135, 647-652. 
Ramírez, M., Ascanio, M., Coronado, M., Gómez, C., Mayorga, L. y Medina, R. (2011). Estilo de vida actual de los pacientes con Diabetes mellitus tipo 2. Revista Ciencia y Cuidado, 8 (1), 21-28.

Secretaría de Salud (2008). Programa de Acción Específico 2007-2012. Diabetes mellitus. México D. F.: Secretaría de Salud en México.

Secretaría de Salud (2010). Acuerdo Nacional para la salud alimentaria estrategia contra el sobrepeso y la obesidad. México D. F.: Secretaría de Salud en México.

Tolosa, A., Candioti, M. y D' Alessandro, M. (2012). Relación entre el estilo de vida y el estado nutricional en pacientes con Diabetes mellitus Tipo 2 de la ciudad de Santa Fe, Argentina. Actualización en Nutrición, 13(3), 170-175.

Unwin, N., Roglic, G. y Whiting, D. (2010). Diabetes tipo 2, su prevención y la Comisión sobre Determinantes Sociales de la Salud de la OMS. Diabetes Voice, 55 (2), 45-47.

Wilson, D. y Ciliska, D. (1984). Life-style assessment: Development and use of the FANTASTIC checklist. Can Fam Physician, 30,1527-1532. 
Anexo 1. Comparación del presente trabajo con otros estudios de estilos de vida en pacientes con Diabetes mellitus tipo 2 evaluados mediante el IMEVID en México.

\begin{tabular}{|c|c|c|c|c|}
\hline $\begin{array}{l}\text { Autores } \\
\text { (año) }\end{array}$ & $\mathbf{n}$ & $\begin{array}{c}\text { Participantes con } \\
\text { Buen Estilo } \\
\text { de vida } \\
\text { \% (Frecuencia) }\end{array}$ & $\begin{array}{c}\text { Participantes } \\
\text { con Inadecuado } \\
\text { Estilo de vida } \\
\% \text { (Frecuencia) }\end{array}$ & Valor de $p$ \\
\hline $\begin{array}{l}\text { Balcázar Nava et al, } \\
(2008)\end{array}$ & 30 & $26.6 \%(8)$ & $73.3 \%(22)$ & $>0.05$ \\
\hline $\begin{array}{l}\text { Corona Meléndez et al, } \\
(2008)\end{array}$ & 43 & $55.8 \%(24)$ & $44.1 \%(19)$ & $<0.05$ \\
\hline $\begin{array}{l}\text { Montejo Briceño } \\
(2009)\end{array}$ & 112 & $42.0 \%(47)$ & $58.0 \%(65)$ & $>0.05$ \\
\hline $\begin{array}{l}\text { Gómez-Aguilar et al, } \\
\text { (2012) }\end{array}$ & 45 & $37.8 \%(17)$ & $62.2 \%(28)$ & $>0.05$ \\
\hline $\begin{array}{l}\text { Tolosa Müller et al } \\
\text { (2012) }\end{array}$ & 50 & $26 \%(13)$ & $74 \%(37)$ & $>0.05$ \\
\hline El presente estudio & 65 & $29.2 \%(19)$ & $70.7 \%(46)$ & \\
\hline
\end{tabular}

